\title{
Jerarquía de ciudades en el Perú con fines de regionalización
}

\author{
Hierarchy of cities in Peru for regionalization purposes
}

\author{
Freddy Arana Velarde $1, a$ \\ https://orcid.org/0000-0003-3439-7491 \\ Rubén Darío Alania Contreras w1,b \\ https://orcid.org/0000-0003-4303-1037
}

\section{Citar como}

Arana Velarde, F., y Alania Contreras, R. (2021). Jerarquía de Ciudades en el Perú con fines de regionalización. Desafíos, 12(1); 16-28. https://doi.org/10.37711/desafios.2021.12.1.257

\section{RESUMEN}

Objetivo. Clasificar jerárquicamente a las ciudades de Perú según su importancia para los procesos de regionalización. Métodos. El estudio fue de enfoque cuantitativo, diseño descriptivo simple; como instrumento de recolección de datos se aplicó una ficha de jerarquía de ciudades que midió las dimensiones tamaño demográfico, tamaño económico y la relevancia urbana a partir de la cual se hizo un análisis factorial de ciudades mayores a 50 mil habitantes, teniendo como muestra 42 ciudades del Perú y excluyéndose a Lima Metropolitana. Resultados. Se halló como ciudades de primera jerarquía: Arequipa en el sur del país, Trujillo, Chiclayo y Piura por el norte, Cuzco al sur oriente y Huancayo al centro; en segunda jerarquía: Tacna, Ica, Chimbote, Iquitos, Pucallpa; tercera jerarquía Ayacucho, Moquegua y Huánuco. Conclusiones. Las ciudades que más influyen para los procesos de regionalización en el nivel macrorregional son: en el norte Trujillo, al sur Arequipa, en el centro Huancayo, en el suroriente Cusco del primer rango jerárquico y en el oriente lquitos del segundo rango.

Palabras clave: jerarquías urbanas; ciudades; regiones; sistema de ciudades.

\begin{abstract}
Objective. To classify hierarchically the cities of Peru according to their importance for the regionalization processes. Methods. The study had a quantitative approach, simple descriptive design; as a data collection instrument, a city hierarchy card was applied, which measured the dimensions of demographic size, economic size and urban relevance; A factor analysis was made of cities with more than 50 thousand inhabitants, taking as a sample 42 cities in Peru, excluding Metropolitan Lima. Results. It was found as cities of first hierarchy: Arequipa in the south of the country, Trujillo, Chiclayo and Piura in the north, Cuzco in the south east and Huancayo in the center; in second hierarchy: Tacna, Ica, Chimbote, Iquitos, Pucallpa; third hierarchy Ayacucho, Moquegua and Huánuco. Conclusions. The cities that most influence the regionalization processes at the macro-regional level are: in the north Trujillo, in the south Arequipa, in the center Huancayo, in the south-east Cusco of the first hierarchical rank and in the east lquitos of the second rank.
\end{abstract}

Keywords: urban hierarchies; cities; regions; system of cities. 


\section{INTRODUCCIÓN}

Sin duda, el centralismo de Lima Metropolitana, capital del Perú, es origen de muchos problemas económicos, sociales, políticos y culturales que obstaculizan el desarrollo del país. De acuerdo con Klauer (2000), el Perú, históricamente centralista, está concentrado casi integramente en Lima; fenómeno que no solo se caracteriza por la mayor concentración de población, sino que también implica la concentración del gasto e inversión, de poder de decisión, de instituciones y empresas, medios de comunicación y especialistas.

Al ser un ente de concentración de factores de desarrollo muy absorbente, la ciudad de Lima desconfigura el proceso natural de desarrollo equilibrado de un sistema de ciudades basado en jerarquías urbanas en el resto del territorio. Dammert (2012) indicó que "Lima se convierte en una cabeza macrocefálica sin que exista una adecuada jerarquía del sistema de ciudades ni entre los ámbitos territoriales. Todas las ciudades del país deberían estar vinculadas, pero existe una profunda distorsión" (p. 68). Ante ese problema se hace indispensable concretar un adecuado proceso de descentralización a través del fortalecimiento de las regiones.

El contexto indica carencias o deficiencias en la planificación del ordenamiento territorial basado en ciudades. En ese sentido, se perciben inadecuadas formas de ocupación del espacio, falta de identificación de los sistemas urbanos y del rol que desempeñan las ciudades en determinados ámbitos del país; entendiendo además que, según las teorías del desarrollo urbano y regional, las relaciones interurbanas basada en jerarquías y funciones urbanas, las ciudades cumplen un determinado rol en el territorio regional y, por ende, constituyen ser ejes ordenadores del territorio en función a ciudades líderes que contribuyan con la formación de regiones (Arana, 2019) y, por qué no, en los procesos de regionalización. Es importante abandonar criterios intuitivos que plantean fusionar departamentos o provincias de manera no técnica, ni científica, criterios que comúnmente se han aplicado en el país.

Partiendo de la propia Constitución Política del Perú (Congreso Constituyente Democrático, 1993), los procesos de regionalización apostaron por la fusión de dos o más departamentos para la formación de macrorregiones. A partir de la cual, se fueron desarrollando experiencias regionalizadoras de orden intuitivo (o experimentos), que no consideraron el rol que desempeñan las ciudades, sus jerarquías, ni el valor socioeconómico, cultural y político que ostentan, no obstante tengan la propiedad de influir sobre vastos territorios, formando regiones (aglomeraciones o sistemas urbanos), gracias al liderazgo que ejercen.

Dichos "experimentos" conllevaron a fracasos consecutivos (fin de los 80's y referéndum 2005); es decir, no fue posible la formación de regiones en el nivel de macrosistemas y, por ende, no se lograron consolidar aglomeraciones integradas ni articuladas con ciudades jerarquizadas basadas en liderazgos e influencias.

Las aglomeraciones, al ser siempre conjuntos urbanos lideradas porciudades más influyentes opor una que ostenta la mayor jerarquía, se constituyen como ejes ordenadores de la formación territorial o de sistemas de ciudades; cualidades que permiten a los territorios ser ordenados y estructurados. Al respecto, Rozenblat et al. (2016), aludiendo a diversos autores (Taylor et al., 2002, 2013; Derudder et al., 2003; Alderson y Beckfield, 2004; Alderson et al., 2010) indicaron que "los análisis previos de la regionalización de las ciudades del mundo funcionaron desde una fuerte suposición a priori de la existencia de un sistema unipolar de ciudades en la globalización" (p. 171); es decir, basados en los campos de fuerza que ejercen las ciudades de mayor jerarquía y que definen su área de influencia.

Un proceso de regionalización basado en la fusión de territorios departamentales es sustantivamente intuitivo, nada técnico ni científico, producto de juegos de intereses políticos o ideológicos que no conducen a resultados favorables. Es indispensable considerar a las ciudades como ejes ordenadores, según su importantica o la influencia que estas ejercen; en otras palabras, entender en términos de Batty (2006) que en una "secuencia del tamaño de ciudades desde las unidades urbanas identificables más pequeñas hasta las más grandes contienen una jerarquía implícita" (p. 1); ellas, las urbes de mayor jerarquía ordenan vastos territorios aglomerando ciudades, formando conjuntos urbanos de acuerdo con su importancia. Esta afirmación fue corroborada por Dickinson en 1961 (como se citó en Contel, 2015) donde indicó que "Las ciudades, en consecuencia, serán consideradas como el "centro [...] rector de la región en donde están ubicadas" (p. 451).

Las teorías del desarrollo urbano y regional contribuyeron sustancialmente en la relevancia de las ciudades para la configuración de regiones; expresiones que vienen desde Von Thünen en 1826 con su teoría de la ubicación, Walter Isard y su teoría de la ciencia regional, en 1956, pasando por August Lösch, Walter Christaller, George K. Zipf y John Q. Stewart, Krueckberg y Silvers, 1978; Potrykowski y Taylor, 1984 (como se citó en Rico Galeana, 2008), 
quienes dieron lugar a la teoría del lugar central (Arana, 2016), entre tantos otros, hasta Paul Krugman con la teoría de la nueva geografía económica en 1991.

Cuanto mayor movimiento económico se despliegue en las ciudades, mayor será su influencia en el territorio regional; como indicó Contel (2015) refiriendo a Walter Christaller que, a mayor complejidad de las actividades económicas en las ciudades, mayor será su posición en el territorio y "mayor será la posición relativa de este lugar central en la jerarquía del sistema de la ciudad” (p. 451).

De manera similar, August Lösch en la misma lógica de Vom Thünen (como se citó en Vergara, 2012), señaló que "la jerarquía urbana está asociada a la ubicación central de la ciudad con respecto de una multiplicidad de centros urbanos" (p. 85); es decir, que un sistema de ciudades configura una región, liderada por una ciudad de mayor jerarquía, gracias a que cumplen mayores funciones urbanas respecto a aquellas de menor relevancia en un territorio.

Es importante destacar que para la formación de regiones es indispensable estructurar un sistema de ciudades basado en aquellas que influyen vastos territorios e intervienen de manera determinante sobre otras de menor rango jerárquico. Al respecto, Santa Cruz (2012), dijo que:

Las regiones tienen como soporte un sistema de ciudades articuladas entre sí. En este sistema se establece una jerarquía y destaca una ciudad principal -hay que recordar la teoría del lugar central- que, por su tamaño de población, nivel de ingresos y concentración de recursos, gravita sobre la dinámica económica de la región. (p. 41)

En el Perú, no se tiene identificado con propiedad los sistemas urbanos, basados en la jerarquización de ciudades, que contribuyan con la organización territorial con fines de regionalización. Es así como el propio Ministerio de Vivienda Construcción y Saneamiento [MVCS] (2006) indicó que "el patrón de asentamiento poblacional desarrollado en el Perú, muestra dos claras tendencias: áreas de alta concentración, con una jerarquía urbana inadecuada, y áreas de alta dispersión" (p. 38). Sin embargo, existe una clasificación del rango de ciudades de manera elemental basada en poblaciones como la del Sistema Nacional de Centros Poblados-SINCEP (Decreto Supremo N. ${ }^{\circ}$ O22-2016-Vivienda, 2016) desde donde "categoriza las ciudades peruanas según su tamaño y su rol funcional en el territorio nacional" (Zucchetti y Freundt, 2019, p. 22); el tamaño entendido como la población residente y el rol funcional como la capitalidad de provincia o departamento. Así están las metrópolis, ciudades mayores, menores, pueblos, villas y caseríos (Decreto Supremo N. ${ }^{\circ} \mathrm{O} 22-$ 2016-Vivienda, 2016).

Esa forma de clasificación es muy básica para establecer jerarquías a pesar de que existen metodologías basadas en las teorías del desarrollo urbano y regional como el modelo gravitacional, el modelo origen destino o el de Gago (2002) basado en el "análisis factorial, mediante el tamaño demográfico, el peso económico, el tamaño del PBI per cápita, la capitalidad nacional y Centralidad aérea internacional" (p.82-83). En el estudio se consideró añadir mayores indicadores, como la capacidad de ingreso de los habitantes, el tamaño de mercado, el nivel de pobreza (índice de desarrollo humano -IDH), entre otros.

El MVCS (2006) define que en el nivel macrorregional se relacionan las características integrales de cada sistema con la jerarquía funcional de sus conglomerados e incluye la importancia económica, tamaño poblacional, patrones de poblamiento, grado de pobreza y niveles de articulación interna.

Estas alternativas de formación de regiones basadas en ciudades no fueron valoradas por el Estado peruano durante los procesos de regionalización, por lo que no se han logrado identificar ciudades estructuradoras de territorios, apoyados en un ranking jerarquizado, que lidere la formación de territorios macrorregionales. Estos vacíos sustantivos fueron motivos suficientes para el inicio de la presente investigación.

Configurada así la situación, con el presente estudio se pretende responder a la siguiente interrogante: ¿de qué manera se encuentran jerarquizadas las ciudades del Perú según su importancia para los procesos de regionalización? Se parte del supuesto de que las ciudades contribuyen de manera muy favorable con la formación y desarrollo de regiones, según su rango jerárquico y grados de influencia en el territorio; así mismo, asumen un determinado liderazgo y se convierten en ejes ordenadores territoriales para los procesos de regionalización en el Perú.

Al respecto, existen algunos antecedente oficiales, como lo establecido por el Decreto Supremo 022-2016-Vivienda (2016) vigente a la fecha, cuyo artículo 9 indica que los "Rangos Jerárquicos de los Centros Poblados del SINCEP", se clasifican por el tamaño de población en metrópoli nacional (la capital de la República), metrópoli regional (capitales de los departamentos), ciudades mayores principales (más a 250 mil hab.), ciudades mayores (de 100 mil a 250 mil hab.), intermedias (de 
20 mil a 50 mil hab.), menores principales (de 10 mil a 20 mil hab.), menores (de 5 mil a 10 mil hab.), villas (2 mil 500 a 5 mil hab.), pueblos (de mil a 2 mil 500 hab.) y caseríos (de 500 a mil hab.). En base a ello tenemos en el artículo 10, que "el centro poblado de mayor jerarquía urbana de cada Unidad Espacial de Planificación Territorial le corresponde la función de centro dinamizador de las actividades económicas dentro de la unidad. De acuerdo a dichos criterios, se estableció en el artículo 11 y cuadro 02, la clasificación demacrosistemas, siendo Limay Callao un gran macrosistema de jerarquía metropolitana nacional; como macrosistema norte las ciudades de Trujillo, Chiclayo, Piura e lquitos en la jerarquía de metrópoli regional; como macrosistema centro, las ciudades de Huancayo y Pucallpa en la jerarquía de metrópoli regional; como macrosistema centrosur, la ciudad del Cusco como metrópoli regional y como macrosistema sur la ciudad de Arequipa en la jerarquía de metrópoli regional.

Desde lo académico, la propuesta de Arana (2019) identificó ciudades con la capacidad de liderar la formación de macrorregiones en el Perú y añadió: "destacan ciudades que logran liderar las aglomeraciones urbanas macrorregionales, formando agrupamientos liderados por Trujillo en el norte, el Centro Huancayo, el Sur Arequipa y el Oriente Iquitos, con el soporte de Tarapoto" (p. 113).

El último Plan Nacional de Desarrollo Urbano 2006-2015 del MVCS (2006) indicó que:

Los cuatro centros urbanos más importantes del país se encuentran en el primer grupo, Lima-Callao y Trujillo en la costa, y Arequipa y Huancayo en la sierra. La primera tiene el rango de metrópoli nacional, mientras las otras tres tienen el rango de metrópolis macrorregional (p. 35).

Por otro lado, existieron estudios que nunca se llevaron a la práctica, tales como:

"Los ocho polos de desarrollo" elaborado por la Oficina Nacional de Planeamiento y UrbanismoONPU- de 1966 basado en la teoría del polo de crecimiento de François Perroux que concluye en 8 ciudades más relevantes del Perú formando regiones en torno a las ciudades de Piura, Chiclayo, Trujillo, Iquitos, Huancayo, Ica, Arequipa, y Cusco (Boissier, 2006).

El Plan Nacional de Desarrollo Urbano de 19741990 de Ministerio de Vivienda y Construcción [MVC] (como se citó en Arana, 2016), basado en el método de las dimensione básicas, determina 5 ciudades del macrosistema urbano nacional como ejes ordenadores territoriales: Trujillo, Iquitos, Huancayo, Arequipa y Lima Metropolitana.
En el presente estudio, para determinar las jerarquías urbanas se acudió a indicadores como el tamaño económico, tamaño demográfico, tamaño de mercado y la relevancia comercial urbana (vuelos comerciales nacionales e internacionales). Basado en Gago (2002), "para la determinación de la jerarquía de las ciudades de América Latina hemos utilizado la combinación de los siguientes indicadores: tamaño demográfico (...), peso económico (...). PBI per cápita (...), capitalidad nacional (...), centralidad aérea internacional" (p. 82-83); a estas dos últimas el presente estudio denomina relevancia urbana.

Esa forma de jerarquizar permite identificar ciudades que cumplen el rol de ejes ordenadores regionales $y$, en distintos niveles subregionales tanto con ciudades de primera, segunda y tercera jerarquía que correspondan a un proceso de regionalización descentralista. De acuerdo con ello, se dividió el estudio en tres partes, siendo: a) tamaño demográfico, b) tamaño de la economía y c) relevancia urbana. Así, la investigación busca brindar un aporte a los procesos de regionalización del país.

El objetivo principal del estudio fue clasificar jerárquicamente a las ciudades de Perú según su importancia para los procesos de regionalización.

\section{MÉTODOS}

\section{Tipo de estudio}

El estudio fue de tipo básico, pues buscó la generación de conocimientos nuevos analizando la variable jerarquía de ciudades; el nivel fue descriptivo y el diseño no experimental, transversal y descriptivo simple. El enfoque fue cuantitativo en el área de urbanismo.

\section{Población y muestra}

Se tuvo como población a las ciudades del Perú; como criterio de selección de todos los centros poblados urbanos y rurales del país se incluyeron aquellos que ostentan la condición de urbana, existiendo a nivel nacional 24 capitales departamentales, 196 provincias y más de 1800 capitales distritales.

Se excluyó a las ciudades con poblaciones menores a 50 mil habitantes según proyecciones al 2020 del Instituto Nacional de Estadística e Informática [INEI] (2018); también se excluyó a la ciudad de Lima Metropolitana debido a que, por sus bastas proporciones, desequilibra al resto del país. El muestreo fue por cuotas, resultando una muestra de 42 ciudades que superan los 50 mil habitantes. 


\section{Instrumentos de recolección de datos}

Se aplicó la ficha de jerarquía de ciudades adaptada de Gago (2002), quien realizó un estudio sobre jerarquía de ciudades en América Latina. El instrumento midió las dimensiones: tamaño demográfico, tamaño económico y la relevancia urbana. Como indicadores: población, tamaño de mercado, producto bruto interno, la capitalidad departamental y vuelos nacionales e internacionales de cada urbe.

La ficha consistió en un cuadro de doble entrada; las primeras columnas fueron destinadas para el listado de ciudades elegidas por macrorregiones y estas, a su vez, por departamentos. Asimismo, dicho cuadro presenta en su primera fila los indicadores como la población de ciudades, el tamaño de mercado, tamaño del PBI, la condición de capitalidad departamental y el transporte aéreo por vuelos anuales, nacionales e internacionales.

La validación del instrumento se realizó mediante el juicio de 10 expertos en desarrollo urbano, resultando en la prueba $\mathrm{V}$ de Aiken entre 0,82 a 0,93; lo que significó una validez muy alta.

\section{Procedimientos de la recolección de datos}

El procedimiento de recolección de datos fue desarrollado del siguiente modo:

a) El tamaño demográfico de cada una de las ciudades se obtuvo del Censo Nacional de Población y Vivienda del año 2017, procesada y publicada por Zucchetti y Freundt (2019), siendo seleccionadas aquellas que superaron los 50 mil habitantes con proyecciones al año 2020; información adquirida del Censo Nacional 2017: XII de Población, VII de Vivienda y III de Comunidades Indígenas (INEI, 2018) y del Primer Reporte Nacional de Indicadores Urbanos 2018 (Zucchetti y Freundt, 2019).

b) El tamaño económico se subdividió en dos aspectos: primero, el tamaño de mercado que consiste en conocer el ingreso per cápita de los habitantes en relación con el tamaño de población de la ciudad; dicha información fue adquirida del INEI (2019). Y segundo, el tamaño del Producto Bruto Interno (PBI) de ciudades, consistente en la relación proporcional entre el PBI per cápita, respecto a la población de la ciudad, según datos del INEI (2020).

c) La relevancia urbana fue medida: primero, por el ejercicio de la capitalidad regional o de departamento, otorgándole un punto a la ciudad capital y cero a las que no tienen dicho rol, de acuerdo con información obtenida del INEI (2018). Segundo, los diversos aspectos comerciales que mantiene una ciudad respecto a otras, tanto en lo nacional como en lo internacional, de acuerdo a la metodología desarrollada por Gago (2002), que se basó en los vuelos comerciales nacionales e internacionales; para el estudio se utilizaron los datos del movimiento general de los aeropuertos, disponibles en la Memoria Anual 2019 de la Corporación Peruana de Aeropuertos [CORPAC] (2020).

\section{Aspectos éticos}

En el estudio se trabajó con datos de acceso público, en el proceso de investigación se mantuvo la fidelidad de los mismos y el tratamiento de la información se realizó con objetividad y se mencionan todas las fuentes utilizadas.

\section{Análisis de datos}

Para el procesamiento de la información se generó una tabla síntesis que muestra en las primeras columnas el listado de las ciudades para un orden jerárquico del 01 al 42 (sin incluir Lima Metropolitana); por el lado horizontal, se mostraron los valores asumidos para cada indicador traducido en índices, lo que posibilitó el hallazgo del promedio ponderado de cada ciudad. Estos valores dieron lugar a la determinación de los niveles jerárquicos, así como los rangos de dichas ciudades, con lo que se estableció el ranking esperado.

Luego se realizó el análisis factorial (Gago, 2002), otorgándole puntaje a cada uno de los indicadores, asignándoles valores para la ponderación, según la relevancia que tienen para los propósitos del estudio; siendo las de mayor puntuación los factores económicos, como el tamaño del PBI (2,5 puntos) y el tamaño de mercado (2 puntos); luego, los vuelos internacionales (2 puntos) y nacionales $(1,5)$, para finalmente el tamaño de la población urbana (1 punto) y la capitalidad regional (1 punto); sumando en total un valor de 10 puntos, por medio de los cuales se obtienen los promedios ponderados que determinarán las jerarquías de las ciudades en análisis.

El resultado del procedimiento presentó el promedio ponderado de índices, con lo que se estableció el orden jerárquico de las 42 ciudades en estudio. El grado de importancia (entre muy alta a muy baja), la influencia de las ciudades (de muy significativa a nada significativa) y la jerarquía o rango jerárquico de la ciudad (de I a la V), se logró en base a la distribución de frecuencia de las ponderaciones, basado en la ley de Sturges (1926). De esa manera fueron determinadas las ciudades ejes, ordenadores potenciales del país con cualidades de liderar la formación de macrorregiones en los procesos de regionalización. 


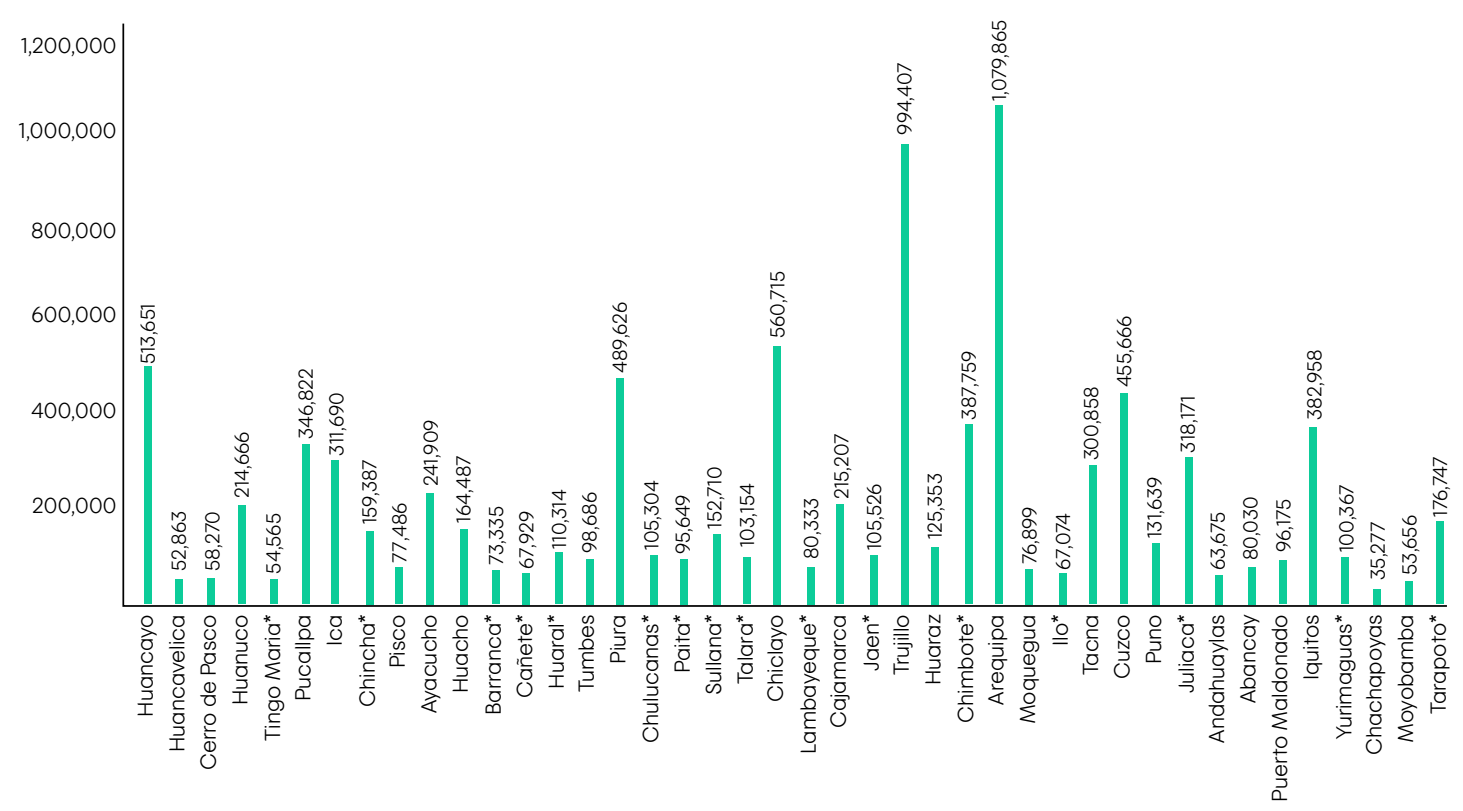

Nota. Elaborada con datos del INEI (2018), Censos Nacionales 2017: XII de Población, VII de Vivienda y III de Comunidades Indígenas y proyecciones al 2020.

Figura 1. Población de ciudades del país mayores a 50 mil hab., 2020

\section{RESULTADOS}

\section{Tamaño demográfico}

\section{Tamaño de la población de ciudades}

Solo una ciudad en estudio superó el millón de habitantes, 4 ciudades superaron el medio millón y 12 ciudades superaron el cuarto de millón: Arequipa, la más poblada, con un millón 79 mil habitantes, seguida por ciudades del norte como Trujillo, con más de 904 mil, Chiclayo con más de 560 mil, luego Huancayo con más de 513 mil, Piura con más de 489 mil, Cuzco con más de 455 mil, Chimbote con más de 387 mil, lquitos con más de 382 mil, Pucallpa con más de 346 mil, Juliaca con más de 318 mil, Ica con más de 311 mil y Tacna con más de 300 mil (ver figuras 1 y 2).

\section{Tamaño de la economía}

\section{Tamaño de mercado de las ciudades}

Las diez ciudades de mayor tamaño de mercado en el país, son fundamentalmente Arequipa, la de
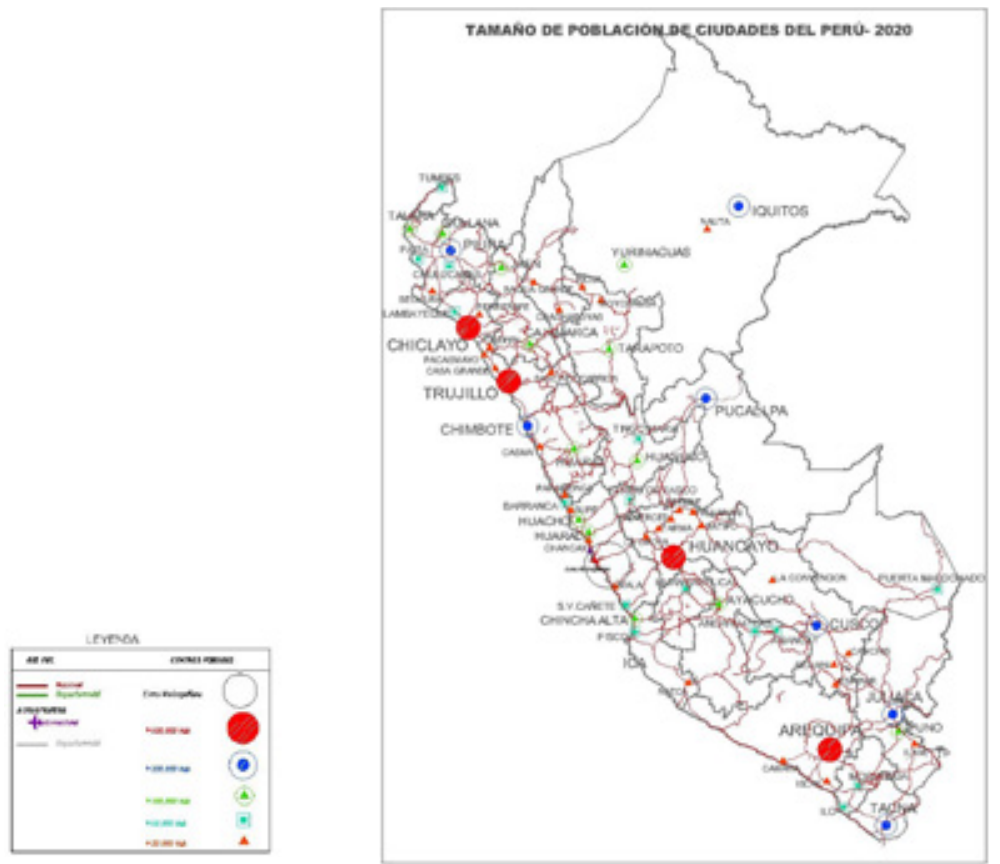

Nota. Elaborada con datos del INEI (2018), Censos Nacionales 2017: XII de Población, VII de Vivienda y III de Comunidades Indígenas y proyecciones al 2020

Figura 2. Tamaño de población de ciudades del Perú mayores a 50 mil hab., 2020 


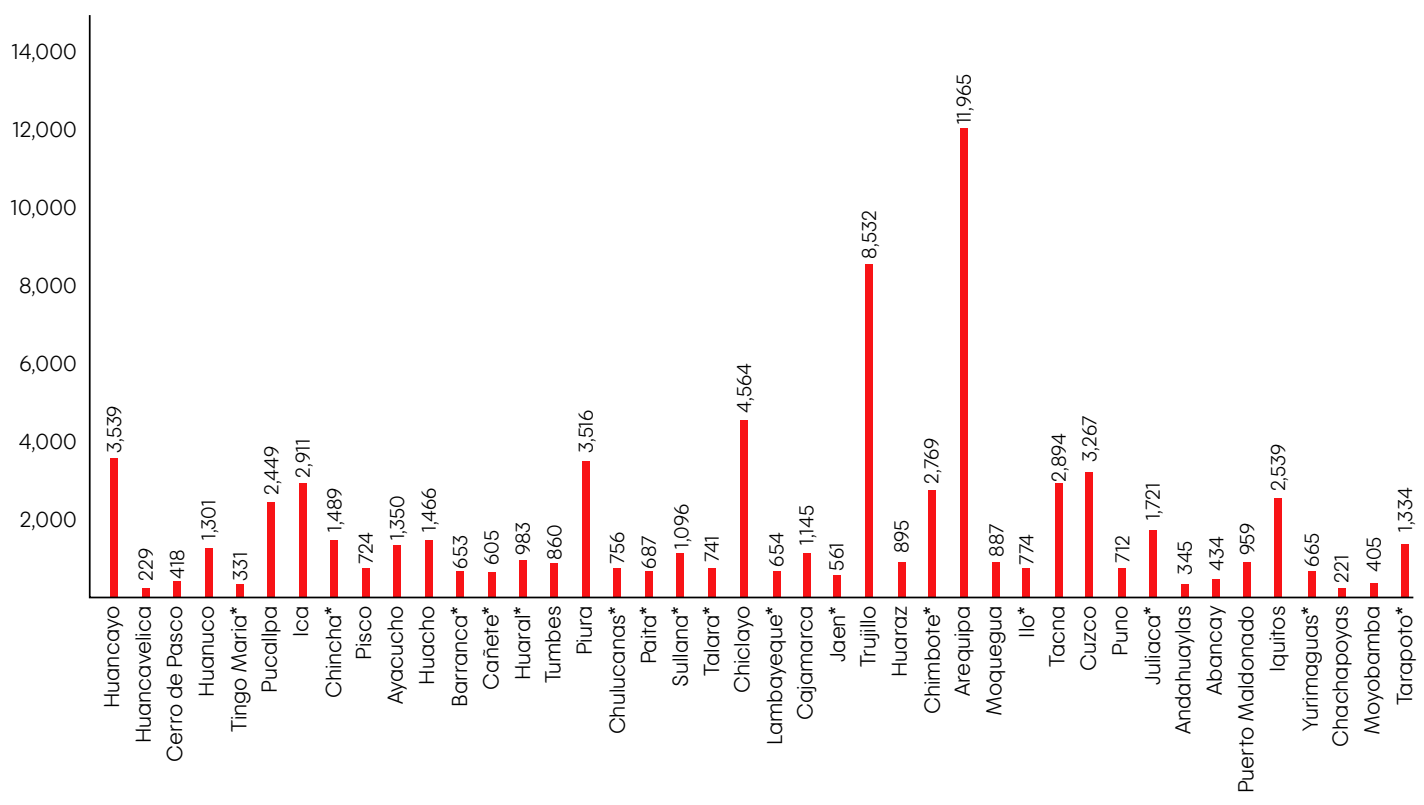

Nota. Elaborada con datos del INEI (2019)

Figura 3. Tamaño de mercado de ciudades del país mayores a 50 mil hab., 2020

mayor potencial, con más de 11965 millones de soles constantes, seguida por ciudades del norte como Trujillo con más de 8532 millones, Chiclayo con 4464 millones, luego Huancayo con 3539 millones, Piura con 3516 millones, Cuzco con 3267 millones, Ica con 2911millones, Tacna con 2894 millones, Chimbote con 2769 millones y, finalmente, lquitos con más de 2539 millones de soles constantes (ver figura 3).

\section{Tamaño del producto bruto interno de las ciudades}

Las 10 ciudades de mayor tamaño de PBI en el país son, fundamentalmente: Arequipa la de mayor potencial con más de 2459528 de miles soles constantes, seguido por Trujillo con 1224 845, Cuzco con 820 512, Chimbote con 741 361, Tacna con 649 811, luego Huancayo con 637 060, Ica con 622 059, Chiclayo con 554 296, Piura con 521 537, finalmente Iquitos con más de 387292 (ver figura 4).

\section{Relevancia urbana de las ciudades del Perú Vuelos anuales nacionales}

Del total de ciudades del país, solo 25 generaron operaciones aéreas en el interior del mismo en el año 2019; de las cuales, las 10 ciudades que tuvieron los mayores movimientos, fueron: Pisco con 79230

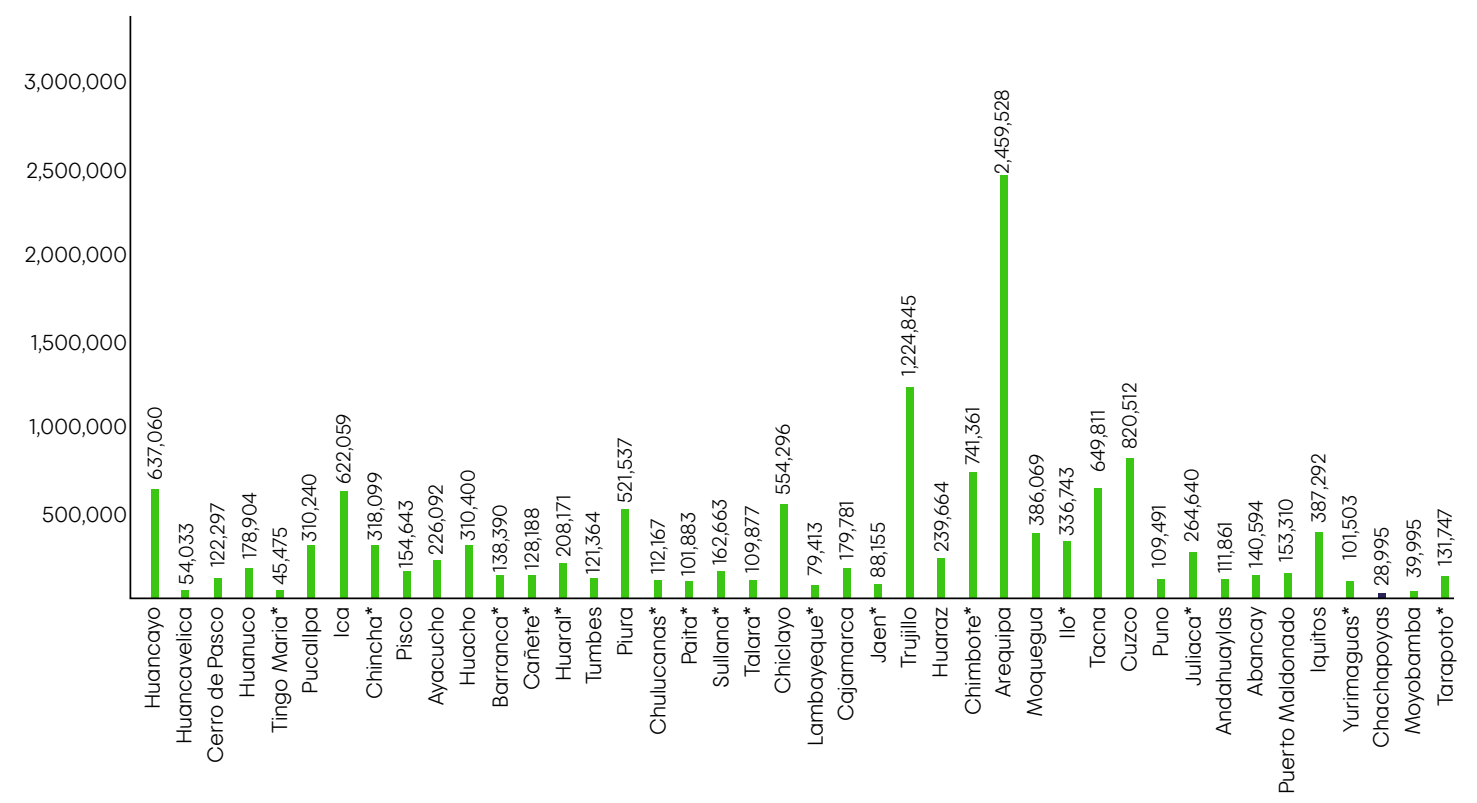

Fuente: Elaboración propia, con datos del INEI (2020).

Figura 4. Tamaño del PBI de ciudades del país mayores a 50 mil hab., 2020 


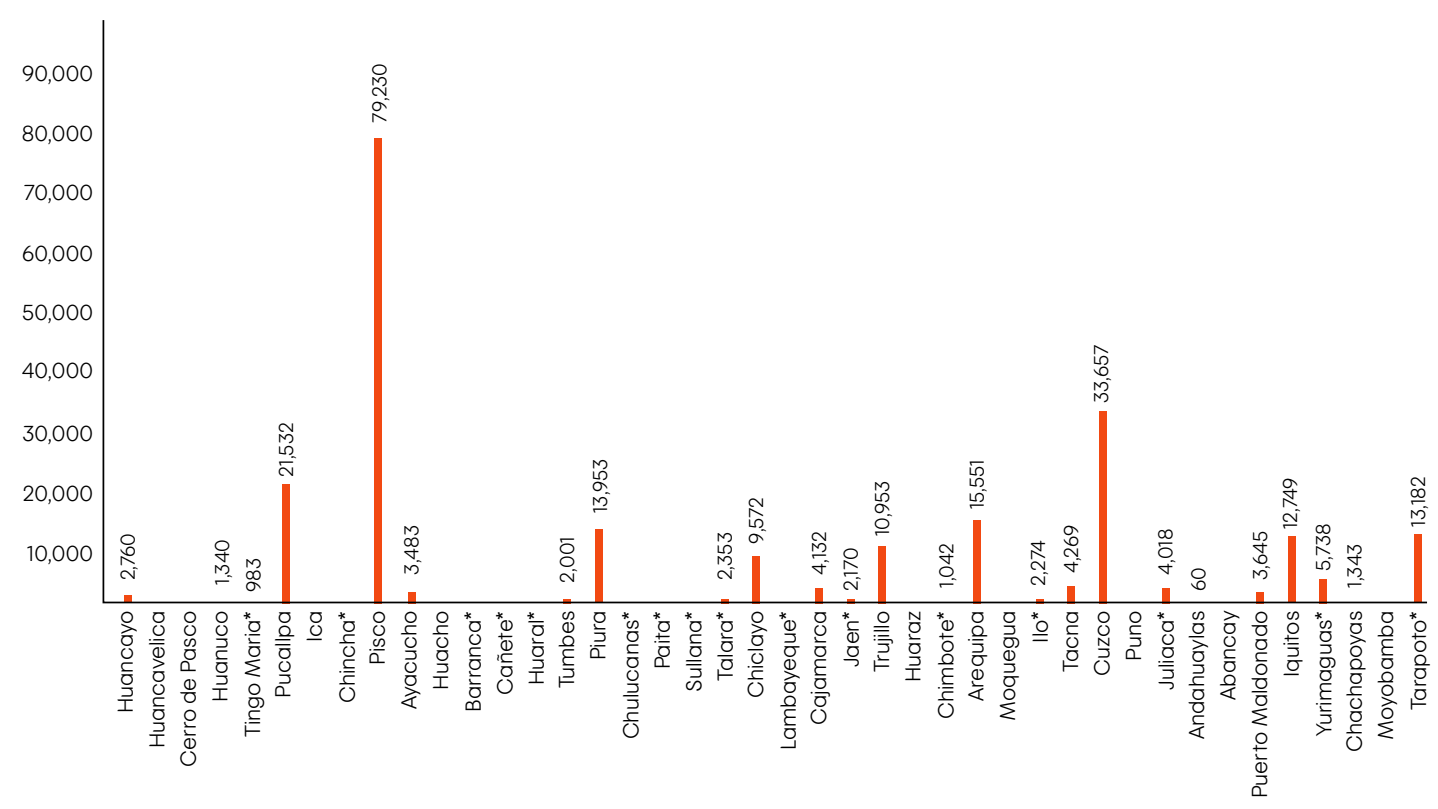

Nota. Elaborada con datos de CORPAC (2020).

Figura 5. Vuelos anuales nacionales en ciudades del país mayores a 50 mil hab., 2019

vuelos anuales, Cuzco como el mayor potencial turístico con 33657 vuelos, seguido por, Pucallpa con 21 532, luego Arequipa con 15 551, Piura con 13 953, Tarapoto con 13 182, Iquitos con 12749 , Trujillo con 10 953, Chiclayo con 9572, finalmente Yurimaguas con 5738 vuelos anuales nacionales (ver figura 5).

\section{Vuelos anuales internacionales}

De las 43 ciudades del país, solo 13 generan operaciones aéreas internacionales; de las cuales, las 05 ciudades de mayor movimiento son: Cuzco como el mayor potencial con 2117 vuelos anuales, seguido por Pisco con 592, Chiclayo con 268, luego Tumbes con 262, finalmente lquitos con 236 vuelos anuales internacionales (ver figuras 6).

\section{Capitalidad departamental de las ciudades}

De las ciudades en análisis, las capitales departamentales son 24; en ellas se desarrollan diversas actividades de orden económico, social, así como las administrativas y es el lugar donde

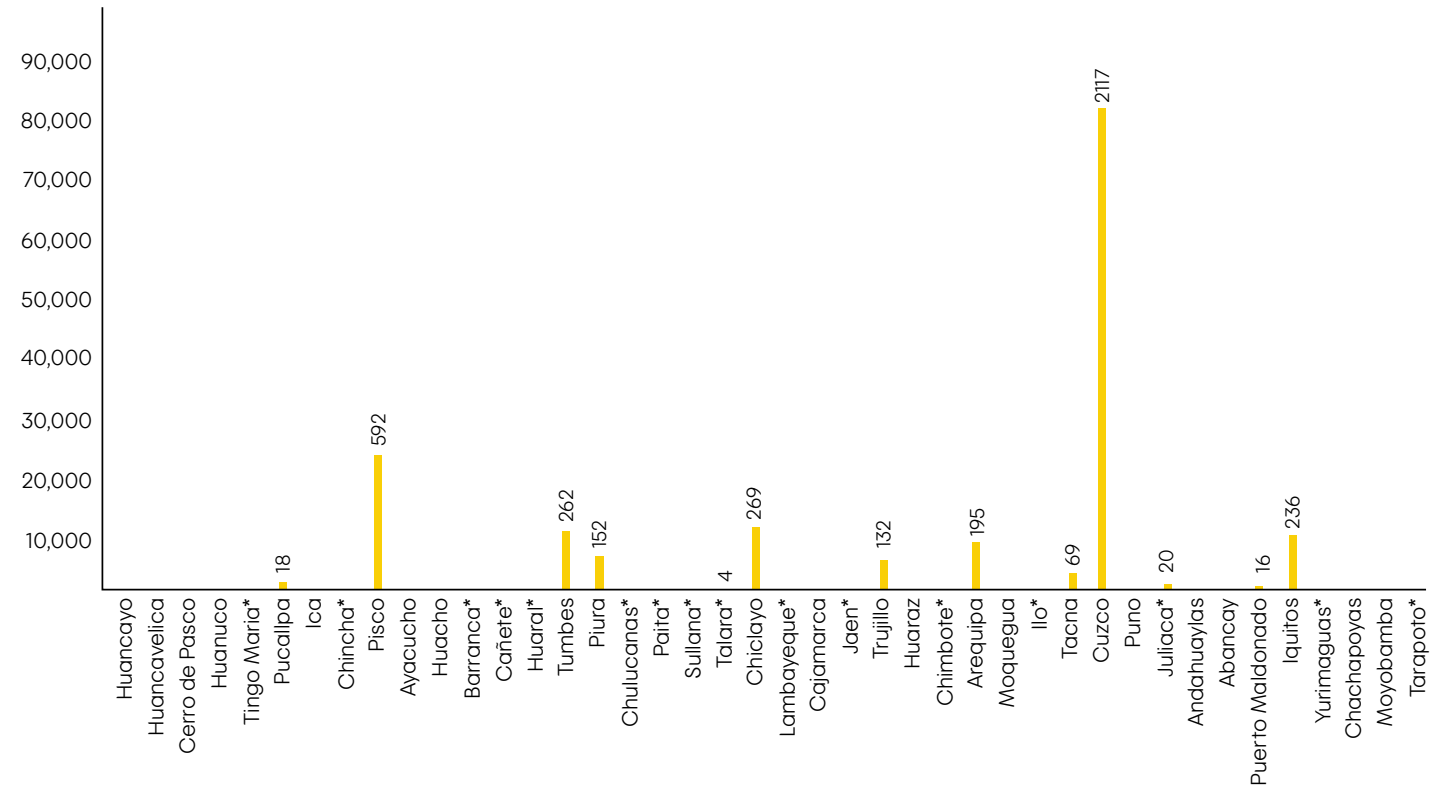

Nota. Elaborado con datos de CORPAC (2020).

Figura 6. Vuelos anuales internacionales en ciudades del país mayores a 50 mil hab., 2019 
se toman decisiones políticas importantes para el desarrollo y que determinan los destinos del ámbito correspondiente. Este es uno de los factores por los que las ciudades cobran mayor importancia, motivos por los que los ciudadanos de las poblaciones periféricas concurren para realizar diversas gestiones, como la consecución de presupuestos y la realización de proyectos y obras en el interior del territorio departamental; además se constituyen como focos de atracción para la migración interna.

\section{Jerarquización de ciudades del Perú}

Los resultados finales del análisis factorial indican que las seis ciudades de mayor rango jerárquico son los siguientes:

Primera jerarquía: Ciudades con importancia muy alta en el país y que influyen de manera muy significativa sobre el territorio: Arequipa en el sur del país, con el mayor promedio ponderado $(9,51$ puntos), seguido por Trujillo que predomina en el

Tabla 1

Jerarquía de ciudades, influencia y rangos por ponderación de índices

\begin{tabular}{|c|c|c|c|c|c|c|}
\hline \multicolumn{7}{|c|}{ Ciudades del país mayores a 50 mil habitantes } \\
\hline $\begin{array}{l}\text { Orden } \\
\text { jerarquico }\end{array}$ & Ciudades & $\begin{array}{c}\text { Promedio ponderado } \\
\text { de indices }\end{array}$ & $\begin{array}{l}\text { Importancia } \\
\text { urbana }\end{array}$ & Influencia de la ciudad & $\begin{array}{l}\text { Jerarquia de la } \\
\text { ciudad }\end{array}$ & $\begin{array}{c}\text { Rango } \\
\text { jerarquico }\end{array}$ \\
\hline 1 & Arequipa & 9,5094 & Muy alta & Muy significativa & Primera & 1 \\
\hline 2 & Trujillo & 6,2020 & Muy alta & Muy significativa & Primera & । \\
\hline 3 & Cuzco & 4,5872 & Muy alta & Muy significativa & Primera & । \\
\hline 4 & Chiclayo & 3,5175 & Muy alta & Muy significativa & Primera & । \\
\hline 5 & Huancayo & 3,1690 & Muy alta & Muy significativa & Primera & । \\
\hline 6 & Piura & 3,0691 & Muy alta & Muy significativa & Primera & I \\
\hline 7 & Tacna & 2,8346 & Alta & Significativa & Segunda & $\|$ \\
\hline 8 & Ica & 2,7413 & Alta & Significativa & Segunda & $\|$ \\
\hline 9 & Chimbote & 2,5061 & Alta & Significativa & Segunda & $\|$ \\
\hline 10 & Iquitos & 2,4837 & Alta & Significativa & Segunda & $\|$ \\
\hline 11 & Pucallpa & 2,2269 & Alta & Significativa & Segunda & $\|$ \\
\hline 12 & Ayacucho & 1,5509 & Media & Regularmente significativa & Tercera & III \\
\hline 13 & Moquegua & 1,5194 & Media & Regularmente significativa & Tercera & III \\
\hline 14 & Huanuco & 1,4106 & Media & Regularmente significativa & Tercera & III \\
\hline 15 & Cajamarca & 1,3874 & Baja & Poco significativa & Cuarta & IV \\
\hline 16 & Pisco & 1,3189 & Baja & Poco significativa & Cuarta & IV \\
\hline 17 & Juliaca* & 1,3149 & Baja & Poco significativa & Cuarta & IV \\
\hline 18 & Huaraz & 1,3082 & Baja & Poco significativa & Cuarta & IV \\
\hline 19 & Tumbes & 1,1964 & Baja & Poco significativa & Cuarta & IV \\
\hline 20 & Puerto Maldonado & 1,1675 & Baja & Poco significativa & Cuarta & IV \\
\hline 21 & Chinhca* & 1,1461 & Baja & Poco significativa & Cuarta & IV \\
\hline 22 & Huacho & 1,1314 & Baja & Poco significativa & Cuarta & IV \\
\hline 23 & Puno & 1,0301 & Baja & Poco significativa & Cuarta & IV \\
\hline 24 & Abancay & 0,9565 & Baja & Poco significativa & Cuarta & IV \\
\hline 25 & $11 o^{*}$ & 0,9027 & Baja & Poco significativa & Cuarta & IV \\
\hline 26 & Cerro de Pasco & 0,8958 & Baja & Poco significativa & Cuarta & IV \\
\hline 27 & Tarapoto* & 0,8643 & Baja & Poco significativa & Cuarta & IV \\
\hline 28 & Huaral $^{*}$ & 0,7588 & Baja & Poco significativa & Cuarta & IV \\
\hline 29 & Sullana* & 0,7521 & Baja & Poco significativa & Cuarta & IV \\
\hline 30 & Moyobamba & 0,7386 & Baja & Poco significativa & Cuarta & IV \\
\hline 31 & Huancavelica & 0,7157 & Baja & Poco significativa & Cuarta & IV \\
\hline 32 & Chachapoyas & 0,6576 & Baja & Poco significativa & Cuarta & IV \\
\hline 33 & Talara* & 0,5240 & Muy baja & Nada significativa & Quinta & V \\
\hline 34 & Chulucanas* & 0,5187 & Muy baja & Nada significativa & Quinta & V \\
\hline 35 & Barranca* & 0,5044 & Muy baja & Nada significativa & Quinta & V \\
\hline 36 & Yurimaguas* & 0,5039 & Muy baja & Nada significativa & Quinta & V \\
\hline 37 & Paita* & 0,4711 & Muy baja & Nada significativa & Quinta & V \\
\hline 38 & Cañete* $^{*}$ & 0,4672 & Muy baja & Nada significativa & Quinta & V \\
\hline 39 & Jaen* & 0,4360 & Muy baja & Nada significativa & Quinta & V \\
\hline 40 & Lambayeque* & 0,4053 & Muy baja & Nada significativa & Quinta & V \\
\hline 41 & Andahuaylas & 0,3635 & Muy baja & Nada significativa & Quinta & V \\
\hline 42 & Tingo Maria* & 0,2353 & Muy baja & Nada significativa & Quinta & V \\
\hline
\end{tabular}




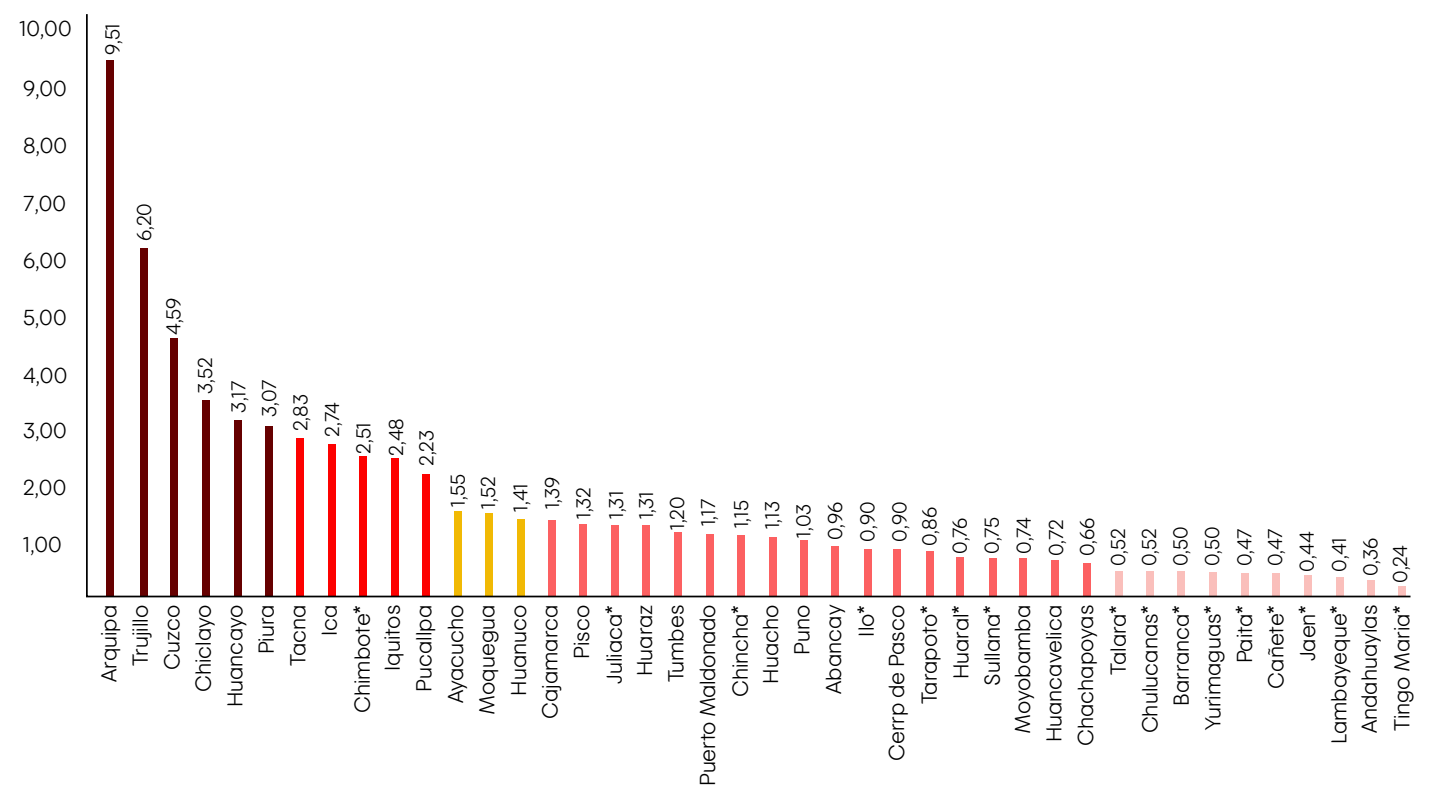

Figura 7. Jerarquía de ciudades en el Perú, por ponderación de índices, 2020

norte $(6,20)$, en tercer lugar, Cuzco en el sur oriente $(4,59)$, luego Chiclayo en el norte $(3,52)$, Huancayo en el centro del país $(3,17)$ y Piura también en el norte con (3,07); son ciudades de primer rango jerárquico, con las que se establecerían los ejes macrorregionales de acuerdo a la configuración territorial que corresponda, durante los procesos re regionalización (ver tabla 1, figuras 7 y 8 ).

Segundajerarquía: Ciudades de alta importancia en el país, que influyen de manera significativa: Tacna en el sur (2,84 puntos), Ica $(2,74)$ y Pucallpa
$(2,23)$ en el centro, Chimbote $(2,5)$ en el al norte e Iquitos $(2,48)$ en el oriente; son ciudades de segundo rango jerárquico que se convierten en ciudades potenciales para estructurar aglomeraciones subregionales, de acuerdo con la configuración territorial que corresponda (ver tabla 1, figuras 7 y 8 ).

Tercera jerarquía: Ayacucho (1,55 puntos), Huánuco $(1,41)$ en el centro, y Moquegua $(1,52)$ en el sur; son ciudades de tercer rango jerárquico con influencia regularmente significativa, de mediana importancia (ver tabla 1, figuras 7 y 8 ).
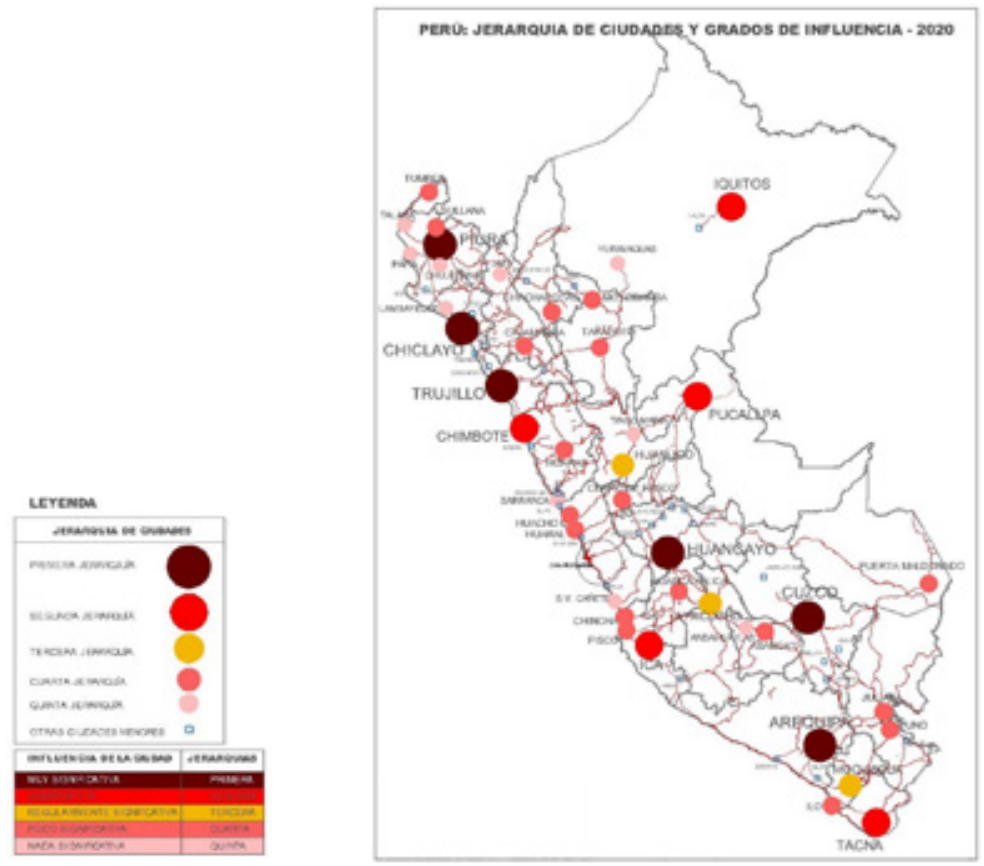

Figura 8. Jerarquía de ciudades y grados de influencia en el territorio nacional, 2020 
Finalmente, las ciudades con nivel bajo o muy bajo de importancia se encuentran en la cuarta y quinta jerarquía, con influencia poco significativa 0 ninguna (ver tabla 1, figuras 7 y 8 ).

Los resultados del estudio presentan datos empíricos que expresan, con claridad, los aportes desarrollados por el estudio. Las unidades de medidas se redactaron de acuerdo con el sistema internacional de unidades (SI).

\section{DISCUSIÓN}

Los resultados del estudio demuestran que las ciudades se encuentran adecuadamente jerarquizadas, con posibilidades de contribuir con la formación y desarrollo de regiones según su rango e importancia; por ende, se convierten en potenciales ejes ordenadores territoriales, para los procesos de regionalización descentralista en el Perú. Las ciudades, según su importancia, de acuerdo con su relevancia urbana, su economía y al tamaño demográfico que muestran los hallazgos, ejercen jerarquías de influencia muy significativa en el territorio, tanto por el norte, centro y sur del país para la formación de macrorregiones, regiones y subregiones.

De los resultados se infiere que la formación de aglomeraciones urbanas o regiones, son efectos de la influencia que ostentan las ciudades de mayor jerarquía configurando un determinado hinterland. Este tipo de ciudades proyectan campos de fuerzas de alta gravitación con condiciones para liderar la regionalización y asumen el rol de ordenadores regionales o subregionales para la nueva configuración territorial del país.

En el caso peruano, de acuerdo con los hallazgos, las ciudades de primera jerarquía que al mismo tiempo son de influencia muy significativa, asumirían la categoría de ejes ordenadores macrorregionales, como: Arequipa en el sur, Trujillo, Chiclayo y Piura por el norte, Cuzco en el sur oriente y Huancayo al centro, con condiciones para liderar un proceso de regionalización en el Perú. Asimismo, las ciudades de segunda jerarquía asumirían el rol de ejes regionales como Tacna, Ica, Chimbote, Iquitos y Pucallpa. En tercera jerarquía se adjudicarían el rol de ejes subregionales ciudades como Ayacucho, Moquegua y Huánuco; en casos muy excepcionales, por la compleja configuración geográfica andina y amazónica del país, algunas ciudades de cuarta jerarquía asumirían dicho rol.

El caso de lquitos es especial; su ubicación, en medio de la Amazonia, imposibilita el acceso por vía terrestre, por lo que las interrelaciones socioeconómicas son por vía aérea o fluvial; esa condición requiere un análisis especial para su ejercicio como eje de ordenamiento macrorregional en el oriente del país, considerando las condiciones imperativas de configuración geográfica del medio.

En el norte del país, existen cuatro ciudades muy relevantes y relativamente muy próximas entre sí, que configuran una gran aglomeración urbana, estableciendo un importante sistema de ciudades, como Trujillo, Chiclayo y Piura en el primer rango jerárquico, y Chimbote del segundo rango; ello demanda realizar estudios que logren determinar un eje ordenador que genere consenso en ese ámbito territorial para estructurar una región única.

Concuerdan en parte con nuestro estudio antecedentes oficiales, como los del Sistema Nacional de Centros Poblados-SINCEP- (vigentes en el país), que son elaborados de manera elemental, basándose en el tamaño de las poblaciones de las ciudades como principal y, prácticamente, el único indicador, que es complementado con el criterio de capitalidad departamental. Ellos presentan, en primera jerarquía, a metrópolis regionales equivalentes al primer rango jerárquico del presente estudio como Arequipa, Trujillo, Cusco, Chiclayo, Huancayo, y Piura; en tanto que, en el segundo rango jerárquico, se ubican las ciudades de Iquitos y Pucallpa; estas últimas, no estarían en el nivel de metrópoli regional como indica el SINCEP, sino como ciudad mayor principal, juntamente con Tacna, Ica y Chimbote, de acuerdo resultados obtenidos por el presente estudio.

Para determinar jerarquías urbanas en el Perú, no basta manejar uno o dos indicadores como el tamaño de la población y la capitalidad departamental. En ese sentido, se discrepa con el SINCEP, entendiendo que las teorías del desarrollo urbano y regional indican que los factores fundamentales para el crecimiento y desarrollo de las ciudades en el territorio son las relaciones interurbanas, con los que se constituyen las grandes aglomeraciones, configurando sistemas de ciudades y, además, formando regiones o subregiones, según el rango jerárquico.

Asimismo, los estudios que anteceden y concuerdan en parte con los resultados obtenidos del presente estudio, son a) El Plan Nacional de Desarrollo Urbano de 1974-1990, del Ministerio de Vivienda y Construcción que nunca fue implementado en el país, y llegó a formular 5 macro sistemas con ciudades como Arequipa, Trujillo, Huancayo, lquitos y Lima como metrópoli nacional y b) El Plan Nacional de Desarrollo Urbano de 2006- 
2015, también del Ministerio de Vivienda, que define tres metrópolis macrorregionales como Arequipa, Trujillo y Huancayo. Las ciudades son los ejes ordenadores fundamentales de configuración de regiones; de acuerdo con sus jerarquías contribuyen favorablemente en los procesos de regionalización, por medio de los cuales se podrán estructurar distintos niveles regionales, ya sean macrosistemas regionales, regiones, subregiones, microregiones, etc. Es decir, en un proceso de tal naturaleza, las ciudades se convierten en una especie de materia prima para la regionalización. Es una opción totalmente distinta a las disposiciones legales del estado peruano que, desde la Constitución Política, disponen la fusión de dos o más departamentos, a las que consideramos como procesos intuitivos empíricos que, además de una alta intromisión de intereses políticos, demostraron ineficiencias en sus procesos e ineficacia como resultados finales.

Según los resultados, existe de manera natural una escala de jerarquías con seis ciudades de importancia urbana muy alta como Arequipa, Trujillo, Cuzco, Chiclayo, Huancayo y Piura, que a la vez son de influencia muy significativa y confirma la jerarquía de ciudades mayores a 50 mil habitantes. También existen ciudades que, por naturaleza propia, no son valoradas por el Estado; estas, a su vez, tienen condiciones muy favorables para los procesos de regionalización en el Perú, confirmando que, al ser del primer rango jerárquico en el país, tendrían las virtudes potenciales para liderar y ejercer como ejes ordenadores de las aglomeraciones del nivel macrorregional o macrosistemas; todo ello gracias a su marcada relevancia urbana, tamaño de su economía y tamaño demográfico de gran importancia en el país.

Asimismo, las ciudades de alta importancia urbana y que a la vez son del segundo rango jerárquico como Tacna, Ica, Iquitos, Chimbote y Pucallpa asumirían el liderazgo de ejes ordenadores regionales (o subregionales) según su emplazamiento en el territorio nacional o, en casos excepcionales como lquitos, actuarían como ejes macrorregionales. En tercera instancia, las ciudades como Ayacucho, Huánuco y Moquegua ejercerían el rol de ordenadores subregionales. El método del análisis factorial utilizado posibilitó el manejo de los indicadores para la obtención de resultados muy claros y los datos utilizados fueron suficientes como para establecer un rango jerárquico adecuado.

El presente estudio se constituye como una línea de base para futuras investigaciones que busquen innovar y esclarecer criterios con fines de la regionalización del país; de este modo, se presentan posibilidades para nuevos estudios basados en modelos como el de origen- destino, el modelo gravitacional o en el análisis factorialregresional. Todos ellos con el fin de contribuir con la modificación de la normativa peruana en lo que corresponde a la descentralización y regionalización, partiendo de la propia Constitución Política del Perú.

\section{Limitaciones}

Una limitación en el estudio fue la falta de antecedentes de investigaciones respecto al tema desarrolladas en el Perú, lo cual se solucionó tomando como referencia estudios realizados en otros países. Otra de las limitaciones fue no tener el acceso a algunos datos oficiales de tipo estadístico a nivel de ciudades, la cual fue resuelta con cálculos de proyecciones al 2020.

\section{Conclusión}

Son las ciudades la materia prima para la regionalización, cuya clasificación según sus jerarquías, propone ejes ordenadores para el país. Estas permitirían estructurar regiones en los distintos niveles, tanto en ámbitos macrorregionales o macrosistemas y subregiones o subsistemas, en concordancia a los rangos jerárquicos, importancia, y niveles de influencia; así como a las condiciones de la configuración geográfica (topográfica) andina y amazónica a las que pertenecen.

Los ejes ordenadores en el nivel macrorregional o macrosistema, en concordancia a los rangos jerárquicos del primer orden de nuestros resultados, vendrían a ser: en el norte Trujillo, al sur Arequipa, en el centro Huancayo, en el sur oriente Cusco y, como caso excepcional por el oriente, lquitos del segundo rango, al no existir en este ámbito otra de mayor jerarquía.

Para la configuración de ejes ordenadores en el nivel regional y subregional, en concordancia a los ámbitos territoriales geográficos, topográficos de la cordillera andina y amazónica, tendríamos: por el norte Chiclayo y Piura del primer rango jerárquico, Chimbote del segundo rango y Cajamarca del cuarto rango; por el sur: Tacna del segundo rango jerárquico, Moquegua del tercer rango y Juliaca del cuarto rango; por el suroriente: Puerto Maldonado y al sur Abancay del cuarto rango; por el centro: Ica y Pucallpa del segundo rango, Ayacucho y Huánuco en el tercer rango; por el oriente, la influencia de las ciudades es baja o poco significativa, siendo las más relevantes Tarapoto y Chachapoyas del cuarto rango.

La escala jerárquica propuesta se constituye como una referencia necesaria para estructurar el ordenamiento territorial con fines de regionalización descentralizada en el Perú. 


\section{REFERÉNCIAS}

Arana, F. (2016). El Rol de las ciudades en la formación y desarrollo de macrorregiones en el Perú [Tesis de maestría, Universidad Nacional de Ingeniería]. Repositorio Institucional de la UNI. http://cybertesis.uni.edu.pe/ handle/uni/6177

Arana, F. (2019). Ciudades para la regionalización. Editorial Académica Española.

Batty, M. (2006). Hierarchy in Cities and City Systems. En D. Pumain (Ed.), Hierarchy in Natural and Social Sciencies (pp. 143- 168). Spinger. http://dx.doi.org/10.1007/14020-4127-6_7

Boissier, S. (2006). Algunas reflexiones para aproximarse al concepto de ciudad-región. Estudios Sociales, 15(28), 165- 168. https://tinyurl.com/y49ym6kr

Congreso Constituyente Democrático. (1993). Constitución Política del Perú. Congreso Constituyente Democrático.

Contel, F. (2015). Concepts of region and regionalization: aspects of its evolution and possible uses to health regionalization. Saude e Sociedade, 24(2), 447- 460 https://doi.org/10.1590/S0104-12902015000200005

CORPAC. (2020). Memoria anual CORPAC 2019 (Informe institucional). CORPAC. http://www.corpac.gob.pe/ Docs/Memorias_CORPAC/Memoria2019.pdf

Dammert, M. (2012). Territorialidad y descentralización de la gestión pública. En T. Cabrera (Comp.), Perú Hoy, Lo urbano en el Perú (pp. 51-75). DESCO. https:// www.desco.org.pe/recursos/site/files/CONTENIDO/22/03_Dammert_Dic_12.pdf

Decreto Supremo N. ${ }^{\circ}$ 022-2016-Vivienda, Reglamento de Acondicionamiento Territorial y Desarrollo Urbano Sostenible (22 de diciembre de 2016). Normas Legales, N. ${ }^{0} 13909$. Diario Oficial El Peruano, 24 de diciembre de 2016.

Gago, C. (2002). Jerarquía urbana en América Latina. NIMBUS, (9-10), 71-97. https://tinyurl.com/y62rkm6t

Instituto Nacional de Estadística e Informática. (2018). Perú Resultados Definitivos (Censos Nacionales 2017: XII de Población, VII de Vivienda y III de Comunidades Indigenas) (Primera ed., Vol. Tomo 1). INEl. https:// www.inei.gob.pe/media/MenuRecursivo/publicaciones_digitales/Est/Lib1544/00TOMO_01.pdf

Instituto Nacional de Estadística e Informática. (2019). Evolución de la pobreza monetaria 2007- 2018 (Informe técnico, Instituto Nacional de Estadística e Informática). INEI.

Instituto Nacional de Estadística e Informática. (2020). PBI de las actividades económicas, por años 2007 - 2019. INEl. https://tinyurl.com/y2ute2q9
Klauer, A. (2000). Descentralización: Sí o sí (2ed.). Autor.

Ministerio de Vivienda Construcción y Saneamiento. (2006). Plan Nacional de Desarrollo Urbano Territorio Para Todos, Lineamientos de Política 2006-2015. MVCS. http://eudora.vivienda.gob.pe/OBSERVATORIO/destacados2/ PlanNacionalDesarrolloUrbano20062015.pdf

Rozenblat, C., Zaidi, F. y Bellwald, A. (2016). The multipolar regionalization of cities in multinational firms' networks. Global Networks, 17(2), 171-194. https://doi.org/10.1111 / glob.12130

Salguero, C. (2006). Enfoques sobre algunas teorías referentes al desarrollo regional [Archivo PDF]. Sociedad Geográfica de Colombia. http://www.economia.unam $\mathrm{mx} /$ academia/inae/pdf/inae5/515.pdf

Santa Cruz, F. (2012). Ciudad, territorio y recursos naturales. En T. Cabrera. (Compiladora), Perú Hoy, Lo urbano en el Perú (pp. 33- 50). DESCO.

Sautu, R., Boniolo, P., Dalle, P. y Rodolfo, E. (2005). Manual de metodología. Construcción del marco teórico, formulación de los objetivos y elección de la metodología. CLACSO. https://tinyurl.com/y4hkasjn

Vergara, R. (2012). Región y ciudades en el sur peruano. En T. Cabrera. (Compiladora), Perú Hoy, Lo urbano en el Perú (pp. 77-101). DESCO.

Zucchetti, A. y Freundt, D. (2019). Ciudades del Perú, Primer Reporte Nacional de Indicadores Urbanos 2018, Con un enfoque de sostenibilidad y resiliencia [Archivo PDF]. PERIFERIA, Territorios Vivos. https:// d2ouvy59p0dg6k.cloudfront.net/downloads/ciudades_sostenibles_1.pdf

\section{Contribución de los autores}

FAV: Planteamiento del proyecto, recolección de datos, análisis de resultados, discusión, revisión final del artículo. RDAC: Análisis de resultados, discusión, revisión final del artículo.

\section{Fuentes de financiamiento.}

La investigación fue realizada con recursos de la Universidad Nacional del Centro del Perú.

\section{Conflictos de interés}

Los autores declaran no tener conflicto de interés.

\section{Correspondencia}

Rubén Darío Alania Contreras

Dirección: Jr. Los Rosales 161 - Departamento 1803, El

Tambo - Huancayo, Perú. CP.: 12006

Cel.: 954681801

Email: ralania@uncp.edu.pe 